H. UEDA

KODAI MATH. J.

6)(1983), 37-46

\title{
A THEOREM ON THE SPREAD RELATION
}

\author{
By Hideharu UEdA
}

\section{Introduction.}

Let $u=u_{1}-u_{2}$ be nonconstant, where $u_{1}$ and $u_{2}$ are subharmonic in the plane C. For such a function $u$, we will write

$$
N(r, u)=\frac{1}{2 \pi} \int_{-\pi}^{+\pi} u\left(r e^{2 \theta}\right) d \theta .
$$

Then the Nevanlinna characteristic of $u=u_{1}-u_{2}$ is defined by

$$
T(r) \equiv T(r, u)=N\left(r, u^{+}\right)+N\left(r, u_{2}\right) .
$$

For $b \in(-\infty,+\infty)$ we define

$$
\sigma_{b}(r, u)=\left|\left\{\theta ; u\left(r e^{\imath \theta}\right)>b\right\}\right| .
$$

(Here, and throughout this note, $|E|$ denotes the one-dimensional Lebesgue measure of the set $E$. Also, $\theta$ is understood to vary between $-\pi$ and $+\pi$.)

In [4], Baernstein proved the following result.

Theorem A. Suppose $u=u_{1}-u_{2}$ is nonconstant, where $u_{1}$ and $u_{2}$ are subharmonic in $\mathbf{C}$. Let $\delta$ and $\lambda$ be numbers satisfying

$$
\lambda>0, \quad 0<\delta \leqq 1, \quad \frac{4}{\lambda} \sin ^{-1}\left(\frac{\delta}{2}\right)^{1 / 2} \leqq 2 \pi .
$$

Assume there exist $r_{0} \geqq 0$ and $b \in(-\infty,+\infty)$ such that $r \geqq r_{0}$ implies

and

$$
N\left(r, u_{2}\right) \leqq(1-\delta) T(r, u)+O(1)
$$

$$
\sigma_{b}(r, u)<\frac{4}{\lambda} \sin ^{-1}\left(\frac{\delta}{2}\right)^{1 / 2}
$$

Then

$$
\lim _{r \rightarrow \infty} \frac{T(r, u)}{r^{2}}=\alpha
$$

Received July 14, 1981 
exists, and is positive or infinite.

Theorem A may be regarded as an analogue of Kjellberg's definitive form [6] of the $\cos \pi \rho$ theorem.

In this note we consider the above result under somewhat weaker assumptions.

Theorem. Let $u, \delta$, and $\lambda$ be as in Theorem $A$ except for the condition (1). Assume instead of (1) that there exists $b \in(-\infty,+\infty)$ such that

$$
m_{l}\left\{r>1 ; \sigma_{b}(r, u) \geqq \frac{4}{\lambda} \sin ^{-1}\left(\frac{\delta}{2}\right)^{1 / 2}\right\}<+\infty .
$$

where $m_{l} E$ denotes the logarithmic measure of the set $E$. Then

$$
\lim _{r \rightarrow \infty} \frac{T(r, u)}{r^{\lambda}}=\alpha
$$

exists, and is positıve or infinite.

From this, we immediately deduce the fellowing

COROLlary. Let $u=u_{1}-u_{2}$, where $u_{1}$ and $u_{2}$ are subharmonic in $\mathbf{C}$, and suppose $u$ has lower order $\mu \in(0, \infty)$. If $\delta(\infty) \equiv \delta(\infty, u)>0$, then for any fixed $b \in(-\infty,+\infty)$ and $\varepsilon>0$,

$$
m_{l}\left\{r>1 ; \sigma_{b}(r, u)>\min \left(2 \pi, \frac{4}{\mu} \sin ^{-1}\left(\frac{\delta(\infty)}{2}\right)^{1 / 2}\right)-\varepsilon\right\}=+\infty
$$

In the above corollary, the quantities $\mu$ and $\delta(\infty)$ ore defined by

$$
\mu=\lim _{r \rightarrow \infty} \frac{\log T(r, u)}{\log r}, \quad \delta(\infty)=1-\varlimsup_{r \rightarrow \infty} \frac{N\left(r, u_{2}\right)}{T(r, u)} .
$$

We remark that this corollary can be deduced also from Theorem 1 in [8].

Without loss of generality, we may prove our theorem under the following additional conditions:

(i) $u_{1}$ and $u_{2}$ are harmonic in a neighborhood of 0 ,

(ii) $b=0$,

(iii) $u_{1}(z) \geqq u_{2}(z)$ for all $z, u_{1}(0)=u_{2}(0)=0$.

For the details, see [4, p89].

\section{Lemmas.}

LeMma 1. ([3]) Let $u=u_{1}-u_{2}$ be nonconstant, where $u_{1}$ and $u_{2}$ a.e subharmonic in $\mathbf{C}$. Put

$$
u^{*}\left(r e^{\imath \theta}\right)=\sup _{E} \frac{1}{2 \pi} \int_{E} u\left(r e^{\imath \omega}\right) d \omega \quad(r>0,0 \leqq \theta \leqq \pi)
$$


where the sup is taken over all sets $E \subset[-\pi,+\pi]$ with $|E|=2 \theta$, and define

$$
u^{\#}\left(r e^{i \theta}\right)=u^{*}\left(r e^{\imath \theta}\right)+N\left(r, u_{2}\right),
$$

Then $u^{\#}(z)$ is subharmonic in the upper half plane.

Lemma 2. ([7], cf. $[5, \S 5]$ ) Let $n$ be a positive integer. Let $\Gamma=\bigcup_{\imath=1}^{n}\left[-r_{\imath}^{\prime},-r_{\imath}\right]$, where $0 \leqq r_{i}<r_{\imath}^{\prime} \leqq r_{\imath+1} \leqq 1,1 \leqq i \leqq n$, and $r_{n+1}=1$. Put $\Gamma^{+}=\{r ;-r \in \Gamma\}$. Let $u$ be subharmonic in the unit disk $\Delta$, and put $m^{*}(r, u)=\inf _{|z|=r} u(z), M(r, u)=\max _{|z|=r} u(z)$, for $0<r<1$. For given $\lambda \in(0,1)$, consider subharmonic functions in $\Delta$ which satisfy

$$
\begin{gathered}
m^{*}(r, u) \leqq \cos \pi \lambda M(r, u) \quad\left(r \in \Gamma^{+}-\{0,1\}\right), \\
u(z) \leqq 1 \quad(z \in \Delta) .
\end{gathered}
$$

For such a fixed $\Gamma$ and $\lambda$, there exists a function $U(z) \equiv U(z, \Gamma, \lambda)$ which has the following properties:

(i) $U$ is bounded, continuous and subharmonic in $\Delta$,

(ii) $U$ is harmonic in $\Delta-\Gamma$,

(iii) $\lim _{z \rightarrow e^{i \theta}} U(z)=1(|\theta|<\pi)$,

(iv) $U(-r)=\cos \pi \lambda U(r)\left(r \in \Gamma^{+}-\{1\}\right)$,

( $\mathrm{v})$ if $u$ is subharmonic in $\Delta$, and if $u$ satisfies (1.1) and (1.2), then $M(r, u)$ $\leqq U(r)$ for $0<r<1$,

(vi) $U$ is the unique function which satisfies (i)-(v),

(vii) if $r \in[0,1)$, then

$$
U(r) \leqq C(\lambda) \exp \left[-\lambda m_{l}\left(\Gamma^{+} \cap[r, 1]\right)\right],
$$

where $C(\lambda)$ is a positvve constant which depends only on $\lambda$.

LEMma 3. ([1]) Let $v$ be subharmonic in $\mathbf{C}$, and suppose that $0<\sigma<1$. If

$$
a=\varliminf_{r \rightarrow \infty} \frac{M(r, v)}{r^{\sigma}}<+\infty \quad\left(M(r, v) \equiv \max _{|z|=r} v(z)\right)
$$

and

$$
\varlimsup_{r_{1}, r_{2} \rightarrow \infty} \int_{r_{1}}^{r_{2}} \frac{m^{*}(r, v)-\cos \pi \sigma M(r, v)}{r^{1+\sigma}} d r \leqq 0 \quad\left(m^{*}(r, v)=\inf _{|z|=r} v(z)\right),
$$

then

$$
\lim _{r \rightarrow \infty} \frac{M(r, v)}{r^{\sigma}}=a
$$

\section{Preliminaries.}

2.1. A function $h(z)$. Set $\beta$ and $\prec$ as follows : 


$$
\beta=\frac{2}{\lambda} \sin ^{-1}\left(\frac{\delta}{2}\right)^{1 / 2}, \quad \gamma=\frac{\beta}{\pi} .
$$

Then

$$
\alpha \equiv \gamma \lambda=\frac{2}{\pi} \sin ^{-1}\left(\frac{\delta}{2}\right)^{1 / 2} \leqq \frac{1}{2}
$$

By assumption, there exists a positive number $A$ such that

$$
N\left(r, u_{2}\right) \leqq(1-\delta) T(r, u)+A \quad(r \geqq 0) .
$$

Since $u(z)$ is nonconstant, $T(r, u) \equiv T(r)$ is unbounded, and so there exists a number $r_{0}>0$ such that

$$
T\left(r_{0}^{\gamma}\right)>\pi A_{1} \equiv \pi A /(1-\cos \pi \alpha) .
$$

Now, Fix $R>2 \max \left(1, r_{0}\right)$ and define

$$
B(t)= \begin{cases}T\left(t^{r}\right) & (0 \leqq t \leqq R) \\ T_{1}\left(R^{r}-\right) & \log \left(\frac{t}{R}\right)+T\left(R^{r}\right) \quad(R \leqq t<\infty),\end{cases}
$$

where $T_{1}\left(t^{r}\right)$ denotes the logarithmic derivative of the function $t \rightarrow T\left(t^{r}\right)$. Then $B(t)$ is a convex increasing function of $\log t$, and the Poisson integral

$$
h(z)=\frac{1}{\pi} \int_{0}^{\infty} \frac{r \sin \theta}{t^{2}+r^{2}+2 t r \cos \theta} B(t) d t \quad\left(z=r e^{\imath \theta}\right)
$$

is harmonic in the slit plane $|\arg z|<\pi$, is zero on the positive axis and tends to $B(r)$ as $\theta \rightarrow \pi-$. Further,

$$
h_{\theta}\left(r e^{\imath \theta}\right)=\frac{1}{\pi} \int_{0}^{\infty} \log \left|1+\frac{r e^{\imath \theta}}{t}\right| d B_{1}(t) \quad(|\theta|<\pi)
$$

and

$$
h_{\theta}(-r) \equiv \lim _{\theta \rightarrow \pi_{-}} \frac{B(r)-h\left(r e^{2 \theta}\right)}{\pi-\theta}=\lim _{\theta \rightarrow \pi_{-}} h_{\theta}\left(r e^{2 \theta}\right)=\frac{1}{\pi} \int_{0}^{\infty} \log \left|1-\frac{r}{t}\right| d B_{1}(t)
$$

hold, where $B_{1}(t)$ is the logarithmic derivative of logarithmically convex nondecreasing function $B(t)$, which were established in $\S 3$ of [2].

By (2.2), (iii) in $\S 0$ and (12) of [2]

$$
\begin{aligned}
\pi h_{\theta}\left(\frac{R}{2}\right) & =\int_{0}^{R} \log \left(1+\frac{R}{2 t}\right) d B_{1}(t) \\
& =B_{1}(R-) \log \frac{3}{2}+\int_{0}^{R} \frac{R / 2}{t+R / 2} d B(t) \\
& =B_{1}(R-) \log \frac{3}{2}+B(R) \frac{1}{3}+\int_{0}^{R} \frac{R / 2}{(t+R / 2)^{2}} B(t) d t
\end{aligned}
$$




$$
\begin{aligned}
& \leqq B_{1}(R-)+B(R) \leqq B(R e)+B(R) \leqq 2 B(R e) \\
& \leqq 2\left\{T_{1}\left(R^{\gamma}-\right)+T\left(R^{\gamma}\right)\right\} \leqq 2\left\{T\left(R^{\gamma} e^{\gamma}\right)+T\left(R^{\gamma}\right)\right\} \leqq 4 T\left(R^{\gamma} e^{\gamma}\right) \text {, i. e. }
\end{aligned}
$$

$$
h_{\theta}\left(\frac{R}{2}\right) \leqq \frac{4}{\pi} T\left(R^{\gamma} e^{r}\right)
$$

Also, for $0<r<R$,

$$
\begin{aligned}
T\left(r^{\gamma}\right)=B(r)= & h(-r)=\int_{0}^{\pi} h_{\theta}(r) d \theta<\pi h_{\theta}(r), \quad \text { i.e. } \\
& h_{\theta}(r)>\frac{1}{\pi} T\left(r^{\gamma}\right) .
\end{aligned}
$$

2.2. A function $h_{1}(z)$. Let $\Delta_{R}=\{z ;|z|<R\}$ and let $h_{1}(z)$ be the bounded harmonic function in $\Delta_{R}$ defined by

$$
\begin{aligned}
h_{1}\left(r e^{\imath \theta}\right)= & \frac{1}{2 \pi} \int_{0}^{\pi} T\left(R^{r}\right) \frac{R^{2}-r^{2}}{R^{2}+r^{2}-2 R r \cos (\theta-t)} d t \\
& +\frac{1}{2 \pi} \int_{\pi}^{2 \pi}\left(-T\left(R^{r}\right)\right) \frac{R^{2}-r^{2}}{R^{2}+r^{2}-2 R r \cos (\theta-t)} d t .
\end{aligned}
$$

Then

$$
\begin{aligned}
\left(h_{1}\right)_{\theta}\left(r e^{2 \theta}\right)= & \frac{1}{2 \pi} \int_{0}^{\pi} T\left(R^{r}\right) \frac{\partial}{\partial \theta}\left(\frac{R^{2}-r^{2}}{R^{2}+r^{2}-2 R r \cos (\theta-t)}\right) d t \\
& +\frac{1}{2 \pi} \int_{\pi}^{2 \pi}\left(-T\left(R^{r}\right)\right) \frac{\partial}{\partial \theta}\left(-\frac{R^{2}-r^{2}}{R^{2}+r^{2}-2 R r \cos (\theta-t)}\right) d t \\
= & \frac{1}{2 \pi} \int_{0}^{\pi}\left(-T\left(R^{r}\right)\right) \frac{\partial}{\partial t}\left(\frac{R^{2}-r^{2}}{R^{2}+r^{2}-2 R r \cos (\theta-t)}\right) d t \\
& +\frac{1}{2 \pi} \int_{\pi}^{2 \pi} T\left(R^{r}\right) \frac{\partial}{\partial t}\left(\frac{R^{2}-r^{2}}{R^{2}+r^{2}-2 R r \cos (\theta-t)}\right) d t \\
= & \frac{T\left(R^{r}\right)}{\pi}\left[\frac{R^{2}-r^{2}}{R^{2}+r^{2}-2 R r \cos \theta}-\frac{R^{2}-r^{2}}{R^{2}+r^{2}+2 R r \cos \theta}\right] \quad(r<R) .
\end{aligned}
$$

Hence $\left(h_{1}\right)_{\theta}(z)$ is also harmonic in $\Delta_{R}$ and

$$
\left(h_{1}\right)_{\theta}\left(-\frac{R}{2}\right)=\frac{8 T\left(R^{r}\right)}{3 \pi} .
$$

2.3. A function $H(z)$. Consider the harmonic function $H(z)$ in $\Delta_{R}^{+}$ $=\left\{z ; z \in \Delta_{R}, \operatorname{Im} z>0\right\}$ defined by

$$
H\left(r e^{\imath \theta}\right)=h\left(r e^{\imath \theta}\right)+\cos \pi \alpha h\left(r e^{\imath(\pi-\theta)}\right)+h_{1}\left(r e^{\imath \theta}\right)+A(\pi-\theta) .
$$

The boundary values of $H$ satisfy 
H. UEDA

$$
\begin{cases}H(-r)=h(-r)=T\left(r^{r}\right) & (0 \leqq r<R), \\ H(r)=\cos \pi \alpha h(-r)+A \pi & (0<r<R), \\ H\left(R e^{\imath \theta}\right) \geqq h_{1}\left(R e^{i \theta}\right)=T\left(R^{r}\right) & (0 \leqq \theta \leqq \pi) .\end{cases}
$$

Now, set

$$
v(z)=u^{\#}\left(z^{\gamma}\right) .
$$

Then by (2.8)

$$
\begin{cases}v(-r)=u^{\#}\left(r^{r} e^{i \beta}\right) \leqq T\left(r^{r}\right)=H(-r) & (0 \leqq r<R), \\ v(r)=u^{\#}\left(r^{r}\right)=N\left(r^{r}, u_{2}\right) \leqq(1-\delta) T\left(r^{r}\right)+A \leqq H(r) & (0<r<R), \\ v\left(R e^{i \theta}\right)=u^{\#}\left(R^{r} e^{i r \theta}\right) \leqq T\left(R^{r}\right) \leqq H\left(R e^{i \theta}\right) & (0 \leqq \theta \leqq \pi) .\end{cases}
$$

Hence, by Lemma 1 and (2.9)

$$
v(z) \leqq H(z) \quad\left(z \in \Delta_{R}^{+}\right) .
$$

\section{Proof of Theorem.}

Set

and

$$
G_{\lambda}=\left\{r>1 ; \sigma_{0}(r, u) \geqq 2 \beta\right\}
$$

$$
F_{\lambda}=(0, \infty)-G_{\lambda} \text {. }
$$

Suppose $r^{\gamma} \in F_{\lambda}$. Since $u \geqq 0$ everywhere, we easily deduce that

$$
v(-r)=u^{\#}\left(r^{r} e^{\imath \beta}\right)=T\left(r^{r}\right) .
$$

It follows from (3.1), (2.9) and (2.10) that for $r^{r} \in F_{\lambda} \cap\left(0, R^{r}\right)$

$$
H_{\theta}(-r) \equiv \lim _{\theta \rightarrow \pi-} \frac{H(-r)-H\left(r e^{i \theta}\right)}{\pi-\theta} \leqq v_{\theta}(-r)=\gamma u_{\theta}^{\#}\left(r^{r} e^{i \beta}\right) .
$$

(Existence of the limit follows from (2.3).) Let $\tilde{u}\left(r e^{i \theta}\right)$ denote the symmetric decreasing rearrangement of $u\left(r e^{i \theta}\right)$ (cf. $[3, \S 3]$ ). Then

$$
u_{\theta}^{\#}\left(r^{r} e^{i \beta}\right)=\tilde{u}\left(r^{r} e^{\imath(\beta-)}\right)=0 \quad\left(r^{r} \in F_{\lambda}\right) .
$$

Hence, by (3.2) and (3.3), $H_{\theta}(-r) \leqq 0$, i.e.

$$
h_{\theta}(-r)+\left(h_{1}\right)_{\theta}(-r) \leqq \cos \pi \alpha h_{\theta}(r)+A \quad\left(r^{r} \in F_{\lambda} \cap\left(0, R^{r}\right)\right) .
$$

If $\alpha<1 / 2$, then by (2.6)

$$
\begin{array}{r}
h_{\theta}(-r)+\left(h_{1}\right)_{\theta}(-r)-A_{1}<\cos \pi \alpha\left[h_{\theta}(r)+\left(h_{1}\right)_{\theta}(r)-A_{1}\right] \\
\left(r^{r} \in F_{\lambda} \cap\left(0, R^{r}\right)\right) .
\end{array}
$$


If $\alpha=1 / 2$, then by assumption $N\left(r, u_{2}\right)$ is bounded. This implies that $u_{2}$ is harmonic, in which case $N\left(r, u_{2}\right)=u_{2}(0)=0$. Hence (2.10) holds with $A=0$. Then, arguing as above, we obtain

$$
h_{\theta}(-r)+\left(h_{1}\right)_{\theta}(-r) \leqq 0 \quad\left(r^{r} \in F_{\lambda} \cap\left(0, R^{r}\right)\right) .
$$

This shows that (3.4) is true also for $\alpha=1 / 2$ with $A_{1}=A$ (an arbitrary positive number).

Here, we consider the functioin $k(z)$ defined by

$$
k(z)=\frac{h_{\theta}(R z / 2)+\left(h_{1}\right)_{\theta}(R z / 2)-A_{1}}{h_{\theta}(R / 2)+\left(h_{1}\right)_{\theta}(R / 2)-A_{1}} \quad(z \in \Delta) .
$$

(Note that the denominator is positive by (2.5) and the choice of $R$.) In view of (2.2), (2.3) and (2.6), $k(z)$ is subharmonic in $\Delta$ and

$$
\left\{\begin{array}{l}
m^{*}(r, k)=\frac{h_{\theta}(-R r / 2)+\left(h_{1}\right)_{\theta}(-R r / 2)-A_{1}}{h_{\theta}(R / 2)+\left(h_{1}\right)_{\theta}(R / 2)-A_{1}}, \\
M(r, k)=\frac{h_{\theta}(R r / 2)+\left(h_{1}\right)_{\theta}(R r / 2)-A_{1}}{h_{\theta}(R / 2)+\left(h_{1}\right)_{\theta}(R / 2)-A_{1}} .
\end{array}\right.
$$

Combining (3.4) and (3.5), we have

$$
m^{*}(r, k)<\cos \pi \alpha M(r, k) \quad\left((R r / 2)^{r} \in F_{\lambda} \cap\left(0,(R / 2)^{\gamma}\right)\right) .
$$

As is easily verified, $m^{*}(r, k)-\cos \pi \alpha M(r, k)$ is upper semicontinuous. Hence

$$
E_{\alpha}=\left\{r \in(0,1) ; m^{*}(r, k)-\cos \pi \alpha M(r, k)<0\right\}
$$

is open. It is clear that

and

$$
m^{*}(r, k) \leqq \cos \pi \alpha M(r, k) \quad\left(r \in E_{\alpha}\right)
$$

$$
k(z) \leqq 1 \quad(z \in \Delta) .
$$

Now, $E_{\alpha}$ is open and so $E_{\alpha}=\bigcup_{n=1}^{\infty}\left(s_{n}, t_{n}\right)$, where $0 \leqq s_{n}<t_{n} \leqq 1$. Here we allow repetition of intervals. Let

$$
T_{j}=\bigcup_{n=1}^{2}\left[s_{n}+\frac{\left(t_{n}-s_{n}\right)}{3 \jmath}, t_{n}-\frac{\left(t_{n}-s_{n}\right)}{3 \jmath}\right] \quad(j=1,2,3, \cdots) .
$$

Then by Lemma 2

$$
M(r, k) \leqq C(\alpha) \exp \left[-\alpha m_{l}\left(T_{j} \cap[r, 1]\right)\right] \quad(j=1,2,3, \cdots) .
$$

Since $m_{l}\left(T_{j} \cap[r, 1]\right) \rightarrow m_{l}\left(E_{\alpha} \cap[r, 1]\right)(\jmath \rightarrow \infty)$, we obtain for $0<r<1$

$$
\frac{h_{\theta}(R r / 2)+\left(h_{1}\right)_{\theta}(R r / 2)-A_{1}}{h_{\theta}(R / 2)+\left(h_{1}\right)_{\theta}(R / 2)-A_{1}} \leqq C(\alpha) \exp \left[-\alpha m_{l}\left(E_{a} \cap[r, 1]\right)\right] \text {. }
$$


H. UEDA

Putting $\widetilde{F}_{\lambda}=\left\{r \in(0,1) ;(\operatorname{Rr} / 2)^{\gamma} \in F_{\lambda}\right\}$, we deduce (3.6) that $\widetilde{F}_{\lambda} \subset E_{\alpha}$, and so by (3.7)

$$
\frac{h_{\theta}(R r / 2)+\left(h_{1}\right)_{\theta}(R r / 2)-A_{1}}{h_{\theta}(R / 2)+\left(h_{1}\right)_{\theta}(R / 2)-A_{1}} \leqq C(\alpha) \exp \left[-\alpha m_{l}\left(\tilde{F}_{\lambda} \cap[r, 1]\right)\right] \quad(0<r<1) .
$$

Hence for $0<r<R / 2$

$$
\begin{aligned}
& \frac{h_{\theta}(r)+\left(h_{1}\right)_{\theta}(r)-A_{1}}{h_{\theta}(R / 2)+\left(h_{1}\right)_{\theta}(R / 2)-A_{1}} \\
& \quad \leqq C(\alpha) \exp \left[-\frac{\alpha}{r} m_{l}\left(F_{\lambda} \cap\left[r^{\gamma},(R / 2)^{r}\right]\right)\right] \\
& \quad=C(\alpha) \exp \left[-\frac{\alpha}{\gamma} \log \left(\frac{R / 2}{r}\right)^{\gamma}+\frac{\alpha}{\gamma} m_{l}\left(G_{\lambda} \cap\left[r^{\gamma},(R / 2)^{r}\right]\right)\right] \\
& \quad=C(\alpha)\left(\frac{r}{R / 2}\right)^{\alpha} \exp \left[\lambda m_{l}\left(G_{\lambda} \cap\left[r^{\gamma},(R / 2)^{r}\right]\right)\right] \\
& \quad \leqq C(\alpha) \exp \left[\lambda m_{l} G_{\lambda}\right] \frac{r^{\alpha}}{(R / 2)^{\alpha}} \equiv B(\alpha) \frac{r^{\alpha}}{(R / 2)^{\alpha}}<+\infty
\end{aligned}
$$

It follows from (2.4)-(2.7), and (3.8) that

$$
\frac{T\left(r^{r}\right) / \pi-A_{1}}{r^{\alpha}}<B(\alpha) \frac{4 T\left(R^{r} e^{r}\right) / \pi+8 T\left(R^{r}\right) / 3 \pi}{(R / 2)^{\alpha}} .
$$

This result may be written

$$
\frac{T\left(r^{r}\right)}{r^{\alpha}}<K_{1} \frac{T\left(R^{r} e^{\gamma}\right)}{(R e)^{\alpha}}+K_{2} r^{-\alpha}
$$

where $K_{1}$ and $K_{2}$ are positive and depend only on $\delta$ and $\lambda$. Replace $r^{r}$ by $r$ and $R^{r} e^{r}$ by $R$. Then we have

$$
\frac{T(r)}{r^{\lambda}}<K_{1} \frac{T(R)}{R^{\lambda}}+K_{2} r^{-\lambda} \quad\left(0<r<\frac{R}{(2 e)^{r}}\right) .
$$

From this, it is easy to see that

$$
\lim _{r \rightarrow \infty} \frac{T(r)}{r^{\lambda}}=+\infty
$$

or

$$
0<\lim _{r \rightarrow \infty} \frac{T(r)}{r^{\lambda}} \leqq \varlimsup_{r \rightarrow \infty} \frac{T(r)}{r^{\lambda}}=\varlimsup_{r \rightarrow \infty} \frac{T\left(r^{r}\right)}{r^{\alpha}}<+\infty
$$

In what follows, we assume (3.9). Since $\alpha \leqq 1 / 2$, the Poisson integral 


$$
I(z)=\frac{1}{\pi} \int_{0}^{\infty} T\left(t^{r}\right) \frac{r \sin \theta}{t^{2}+r^{2}+2 t r \cos \theta} d t
$$

is a positive harmonic function in the upper half plane, with boundary values $I(-r)=T\left(r^{r}\right), I(r)=0$ for $r \geqq 0$. Then, arguing as in 2.3., we have

$$
v\left(r e^{i \theta}\right) \leqq I\left(r e^{i \theta}\right)+\cos \pi \alpha I\left(r e^{2(\pi-\theta)}\right)+A(\pi-\theta)
$$

on the real axis. Since $v\left(r e^{i \theta}\right) \leqq T\left(r^{\gamma}\right)=O\left(r^{\alpha}\right)<o(r)$, the above inequality holds throughout the upper halp plane. Also equality holds for $r^{\gamma} \in F^{2}$ and $\theta=\pi$. Hence

so that

$$
I_{\theta}(-r)-\cos \pi \alpha I_{\theta}(r)-A \leqq v_{\theta}(-r)=0 \quad\left(r^{r} \in F_{\lambda}\right),
$$

$$
I_{\theta}(-r)-A_{1} \leqq \cos \pi \alpha\left[I_{\theta}(r)-A_{1}\right] \quad\left(r^{\gamma} \in F_{\lambda}\right) .
$$

$$
\pi I_{\theta}(r)=\int_{0}^{\infty} \log \left(1+\frac{r}{t}\right) d T_{1}\left(t^{r}\right)=\int_{0}^{\infty} \frac{r}{t+r} d T\left(t^{r}\right)=\int_{0}^{\infty} \frac{r T\left(t^{r}\right)}{(t+r)^{2}} d t
$$

and so by (3.9)

$$
0<\varliminf_{r \rightarrow \infty} \frac{I_{\theta}(r)}{r^{\alpha}} \leqq \varlimsup_{r \rightarrow \infty} \frac{I_{\theta}(r)}{r^{\alpha}}<+\infty .
$$

If we put $J(z)=I_{\theta}(z)-A_{1}$ and

$$
E_{\alpha}^{\prime}=\left\{r ; m^{*}(r, J)-\cos \pi \alpha M(r, J)>0\right\},
$$

then by (3.11) and (3.13)

$$
\begin{gathered}
\int_{1}^{\infty} \frac{\left[m^{*}(r, J)-\cos \pi \alpha M(r, J)\right]^{+}}{r^{1+\alpha}} d r=\int_{E_{\alpha}^{\prime} \cap(1, \infty)} \frac{m^{*}(r, J)-\cos \pi \alpha M(r, J)}{r^{1+\alpha}} d r \\
\quad<(1-\cos \pi \alpha) \int_{E_{\alpha}^{\prime} \cap(1, \infty)} \frac{M(r, J)}{r^{1+\alpha}} d r \\
\quad=(1-\cos \pi \alpha) \int_{E_{\alpha}^{\prime} \cap(1, \infty)} \frac{O\left(r^{\alpha}\right)}{r^{1+\alpha}} d r=O\left(m_{l} E_{\alpha}^{\prime}\right)<+\infty .
\end{gathered}
$$

Hence

$$
\varlimsup_{r_{1}, r_{2} \rightarrow \infty} \int_{r_{1}}^{r_{2}} \frac{\left[m^{*}(r, J)-\cos \pi \alpha M(r, J)\right]}{r^{1+\alpha}} d r \leqq 0 .
$$

Using Lemma 3, we deduce from (3.13) and (3.14) that

$$
\begin{gathered}
\lim _{r \rightarrow \infty} \frac{M(r, J)}{r^{\alpha}}=a \quad(0<a<+\infty), \text { i.e. } \\
\lim _{r \rightarrow \infty} \frac{I_{\theta}(r)}{r^{\alpha}}=a
\end{gathered}
$$


By (3.12)

$$
I_{\theta}(r)=\frac{1}{\pi} \int_{0}^{\infty} T\left(t^{r}\right) \frac{r}{(t+r)^{2}} d t=g * K(r),
$$

where $g(t)=T\left(t^{r}\right), K(t)=\frac{1}{\pi} \frac{t}{(1+t)^{2}}$. Using Lemma 4 of [4], we deduce from (3.15) and (3.16) that

$$
\lim _{r \rightarrow \infty} \frac{T(r)}{r^{\lambda}}=\lim _{r \rightarrow \infty} \frac{T\left(r^{r}\right)}{r^{\alpha}}=\frac{\sin \pi \alpha}{\alpha} a .
$$

This completes the proof of our theorem.

\section{REFERENCES}

[1] J.M. Anderson, Asymptotic properties of integral functions of genus zero, Quart. J. Math. Oxford Ser. (2) 16 (1965), 151-165.

[2] A. Baernstein II, A generalization of the $\cos \pi \rho$ theorem, Trans. Amer. Math. Soc. Vol. 193 (1974), 181-197.

[3] A. Baernstein II, Integral means, univalent functions and circular symmetrization, Acta Math. 133 (1974), 139-169.

[4] A. Baernstein II, Regularity theorems associated with the spread relation, J Analyse Math. Vol. 31 (1977), 76-111.

[5] M. Essén, The $\cos \pi \lambda$ theorem, Lecture notes in Mathematics 467 Springer Verlag (1975).

[6] B. KJellberg, A theorem on the minimum modulus of entire functions, Math. Scand. 12 (1963), 5-11.

[7] $\mathrm{Z}$ J. LEwis, Some theorems on the $\cos \pi \lambda$ inequality, Trans. Amer. Math. Soc Vol. 167 (1972), 171-189.

[8] H. UEDA, Two results associated with spread relation, Kodai Math. J. Vol. 5 No. 1 (1982), 89-99.

Department of Mathematics

Daido Institute of TeChNology,

Daido-cho, MinAmi-KU, NAgOYA, JAPAN 